\section{Response to: 'Antinuclear antibodies: mitotic patterns and their clinical associations' by Betancur and Gómez-Puerta}

It is very much appreciated that the correspondence of Betancur and Gómez-Puerta ${ }^{1}$ calls for attention for the clinical relevance of mitotic patterns observed in HEp-2 indirect immunofluorescence assays (IIFA). Indeed, in the recent publication of the International Consensus on ANA Patterns (ICAP) consortium, it is concluded that the clinical relevance of the mitotic patterns is limited. ${ }^{2}$ With exception of the Nuclear Mitotic Apparatus (NuMA)-like pattern (anti-cellular (AC)-26), there is not much available evidence that these patterns clearly hint at a particular clinical manifestation. For the NuMA-like pattern, there is formal evidence that approximately one-half of the patients have clinical features of a systemic autoimmune disease, especially Sjögren's syndrome, polymyalgia rheumatica, systemic lupus erythematosus, polymyositis and autoimmune hepatitis. ${ }^{3}$ ICAP initially defined 28 patterns ordered in a classification tree based on nuclear, cytoplasmic or mitotic reactivity of autoantibodies on HEp-2 cells. ${ }^{4}$ Subsequently, the nuclear Topo I-like pattern (AC-29) was added, ${ }^{5}$ and the codes AC-0 and AC-XX were given to negative results or undefined patterns, respectively. ${ }^{6}$ The international consensus, as defined in the classification tree, the nomenclature and the pattern definitions enable harmonisation in HEp-2 IIFA pattern assignment. This harmonisation is crucial in eventually finding clinical associations of rare patterns. The original study of Betancur et al,,$^{7}$ unfortunately, does not follow the international consensus. While nuclear and cytoplasmic patterns are equally identified, Betancur $e t$ al utilise a cell-cycle related or mitotic spindle apparatus (MSA) category instead of mitotic patterns. As such, and differently from the ICAP approach, the centromere protein (CENP)-F-like pattern (AC-14) is not considered a nuclear pattern, but an MSA-pattern. Also, the mitotic pattern referred to by ICAP as 'intercellular bridge' (AC-27) is assigned as 'midbody'. The lack of alignment with ICAP, however, is to be explained by the fact that the study of Betancur et al is a retrospective study based on data obtained in 2013 and 2014, that is, before the first ICAP publication. ${ }^{4}$

The study of Betancur $e t a l^{6}$ is based on a very large, real-life cohort of 113491 consecutive HEp-2 IIFA test results. Such large studies are mandatory to identify possible clinical associations of the rare mitotic patterns. The design of the study also illustrates the difficulty of adequately defining clinical associations. First, as the authors noted themselves, the study cohort is characterised by a very high positivity rate: $53 \%$ of all the tests were positive. There may be different explanations for this finding, which is in sharp contrast with our own study revealing only $16 \%$ positive results, ${ }^{8}$ but by including consecutive samples, it is to be expected that follow-up samples of the same patients were included. Second, in the positive cohort, $0.46 \%$, that is, 278 sera, revealed a NuMA-like MSA pattern. For 152 of these samples, a complete medical history was available and for 65 samples, a final diagnosis was obtained. Only the latter were included in the study for further analysis. The other sera were excluded because the respective patients did not meet the clinical criteria of an autoimmune disease or because they were considered false positive. Obviously, this resulted in some kind of selection bias. Moreover, as a false positive result is to be considered a clinically unexpected positive finding, the exclusion of such results prevents the identification of unexpected associations. Third, the presented relative risks of anti-MSA antibodies for autoimmune diseases, for instance, the centrosome pattern (AC-24) for undifferentiated connective tissue disease (13.58 (3.88-47.46)) or the intercellular-bridge/midbody pattern (AC-27) for sensorineural hearing loss (15.76 (3.70-67.09)), indicate a rather strong association. The high confidence intervals, however, suggest that the relative risks are calculated within the selected cohort of $116 \mathrm{sera} /$ patients. Obviously, the relative risk would have been much more realistic if calculated based on all mitotic patterns identified and on all clinical manifestations in the total cohort.

Altogether, it is evident that identifying possible clinical associations for the rare mitotic patterns, as well as for rare cytoplasmic patterns, is extremely difficult. This topic has been on the agenda of the ICAP executive board, and a structured project with a clear study design is in the pipeline. It is obvious that data should be obtained in a prospective study including consecutive patients that at the time of first testing revealed a mitotic HEp-2 IIFA pattern. For each patient, a list of clinical manifestations related to the suspected and/or underlying disease should be generated. It should be a multicenter study to warrant the inclusion of sufficient patients and to cover the distinct ethnic populations worldwide. The pattern assignment should be according to pre-set definitions, preferentially according to ICAP, and should not be influenced by the brand of HEp-2 slides used. As far as possible, it is also important to test the sera for antigen-specificity because intermingling autoantibodies revealing a similar HEp-2 IIFA pattern, but being reactive to distinct antigens, may eventually hamper the finding of a clear-cut clinical association. In the end, we fully agree with the final statement of Betancur and Goméz-Puerta that 'there remains much work still to be carried out in this expanding field'. A collaborative effort is the only way to reach the final goal.

\section{Jan Damoiseaux $\odot,{ }^{1}$ Luis Eduardo Coelho Andrade ${ }^{2}$ \\ ${ }^{1}$ Maastricht University Medical Center, Maastricht, The Netherlands \\ ${ }^{2}$ Rheumatology Division, Universidade Federal de Sao Paulo, Sao Paulo, Brazil}

Correspondence to Dr Jan Damoiseaux, Maastricht University Medical Center, Maastricht 6229 HX, The Netherlands; jan.damoiseaux@mumc.nl

Handling editor Josef S Smolen

Competing interests None declared.

Patient consent for publication Not required.

Provenance and peer review Commissioned; internally peer reviewed.

(C) Author(s) (or their employer(s)) 2020. No commercial re-use. See rights and permissions. Published by BMJ.

\section{Check for updates}

To cite Damoiseaux J, Andrade LEC. Ann Rheum Dis 2020;79:e64.

Received 5 April 2019

Accepted 7 April 2019

Published Online First 29 April 2019

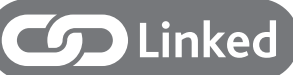

- https://doi.org/10.1136/annrheumdis-2019-215428

Ann Rheum Dis 2020;79:e64. doi:10.1136/annrheumdis-2019-215439

ORCID iD

Jan Damoiseaux http://orcid.org/0000-0003-4007-6985

\section{REFERENCES}

1 Betancur JF, Gómez-Puerta JA. Antinuclear antibodies mitotic patterns and their clinical associations. Ann Rheum Dis 2020;79:e63.

2 Damoiseaux J, Andrade LEC, Carballo OG, et al. Clinical relevance of HEp-2 indirect immunofluorescent patterns: the International consensus on ANA patterns (ICAP) perspective. Ann Rheum Dis 2020;79:879-89. 
3 Andrade LE, Chan EK, Peebles $\mathrm{CL}$, et al. Two major autoantigen-antibody systems of the mitotic spindle apparatus. Arthritis Rheum 1996;39:1643-53.

4 Chan EKL, Damoiseaux J, Carballo OG, et al. Report of the first international consensus on standardized Nomenclature of antinuclear antibody HEp-2 cell patterns 2014-2015. Front Immunol 2015;6.

5 Andrade LEC, Klotz W, Herold M, et al. International consensus on antinuclear antibody patterns: definition of the ac-29 pattern associated with antibodies to DNA topoisomerase I. Clin Chem Lab Med 2018;56:1783-8.
6 Herold M, Klotz W, Andrade LEC, et al. International consensus on antinuclear antibody patterns: defining negative results and reporting unidentified patterns. Clin Chem Lab Med 2018;56:1799-802.

7 Betancur JF, Londoño A, Estrada VE, et al. Uncommon patterns of antinuclear antibodies recognizing mitotic spindle apparatus antigens and clinical associations. Medicine 2018;97.

8 Avery TY, van de Cruys M, Austen J, et al. Anti-nuclear antibodies in daily clinical practice: prevalence in primary, secondary, and tertiary care. $J$ Immunol Res 2014;2014:1-8. 